\title{
Effect of CS preexposure on the conditioned ejaculatory preference of the male rat: behavioral analyses and neural correlates
}

\author{
Gonzalo R. Quintana, Misha Jackson, Mojdeh Nasr, and James G. Pfaus \\ CSBN/Psychology, Concordia University, Montréal, Quebec H4B 1R6, Canada
}

\begin{abstract}
Early experiences with sexual reward play a pivotal role in the formation of sexual behavior and partner preference. Associations of salient partner cues, or even neutral cues on a partner, with sexual reward states are a product of Pavlovian learning. However, the extent to which first experiences that associate a neutral stimulus with no immediate consequence, and how that association may affect subsequent associability after being paired with a sexual reward state after copulation to ejaculation, remains unclear. To address this question, sexually naïve males were preexposed over one or five trials to almond scented gauze pads prior to training during which half of the males were trained 10 times with scented receptive females, and the other half with unscented receptive females. A final test of partner preference was conducted in a large open field containing two sexually receptive females, one scented and the other unscented. Males developed a conditioned ejaculatory preference for the type of female they were trained with, except when they were preexposed five times to the odor and then trained with females bearing the same odor, indicating a significant CS preexposure effect. One CS preexposure was not sufficient to inhibit subsequent conditioning. Exposure to the scent before perfusion for inmunohistochemistry, revealed different patterns of brain activation in brain areas previously associated with the development of partner preference, like the medial preoptic area, ventral tegmental area, nucleus accumbens, basolateral amygdala, among others, depending on group membership. Thus, CS preexposure results in a subsequent impairment of the association that links the odor cue to sexual reward and preference. This highlights the impact of the first sexual experiences in future partner preference.
\end{abstract}

Latent inhibition is the phenomenon in which the preexposure to a conditional stimulus (CS) leads to a disruption or retardation of a subsequent trained association with the same CS (e.g., Lubow and Moore 1959). For example, animals preexposed to a saline solution used later on to train conditioned taste aversion showed retardation of the association in comparison to a control group who was not exposed to it previously (Rodríguez and Alonso 2002). Most theories coincide in that latent inhibition is the result of a reduction in associability or attention to CS during preexposure (Schmajuk 2002). Whether by hindering the storage or retrieval of the association, how this process occurs is still a matter of debate (Schmajuk 2002).

Evidence shows that latent inhibition is a context-specific phenomenon (McLaren et al. 1994), that also varies depending on the CS duration (Westbrook et al. 1981), CS intensity (Schnur and Lubow 1976), numbers of trials of preexposure (Lantz 1973), total CS preexposure time (Ayres et al. 1992), inter-trial interval (Lantz 1973), among others. This "latent" learning phenomenon and its properties highlights the ability of animals to form new associations through passive, nonreinforced preexposure of CS's, demonstrating that previous experiences influence when trained to learn new associations with neutral cues. This can have important implication for learning phenomena experienced further on in the life of animals when they reach a certain level of biological maturity, like sexual behavior.

Animals become sexually active after reaching puberty (Hull and Rodriguez-Manzo 2009). At this point, most have already been preexposed to several classes of stimuli that can be contingent when mating. Zamble et al. (1986) demonstrated that single CS or contextual cues can facilitate copulation in Japanese quail (i.e., re-

\section{Corresponding author: jim.pfaus@concordia.ca}

Article is online at http://www.learnmem.org/cgi/doi/10.1101//m.048108. 118. duced ejaculation latency) if they predicted copulation with a receptive female. However, when animals were preexposed enough times to the mating context, the background cues became latently inhibited. Particularly, when studying how animals choose a sexual partner, the literature has confirmed that early experiences crystallize not only sexual behaviors and responses, but also place and partner preferences (Kippin et al. 1998; Parédes and Vasquez 1999; Tenk et al. 2009). For example, it has been shown that animals will choose a partner that resembles more an adoptive mother than the genetic mother (Kendrick et al. 1998). In that experiment, Kendrick and colleagues separated male and female sheep and goats and cross-fostered them. These animals were allowed to engage in social contact with members of their genetic species during development. When animals reached adulthood, they were tested for social and mate preference between members of their own and foster species. Results showed that both cross-fostered males and females significantly chose to socialize and selectively mate more with partners of their nongenetic species. These effects were more pronounced and long-lasting in males than in females. In contrast, all control animals preferred to socialize and mate exclusively with members of their own genetic species (Kendrick et al. 1998). Likewise, Fillion and Blass (1986) demonstrated that males exposed early on to a neutral lemon odor cue, ejaculated more readily with females bearing the same cue. In their experiment, male pups were exposed from day 2 until separation (day 28) to dams whose vaginal area and nipples were scented with a lemon odor. Subsequently, between days 90 and 120, males were

(C) 2018 Quintana et al. This article is distributed exclusively by Cold Spring Harbor Laboratory Press for the first 12 months after the full-issue publication date (see http://learnmem.cshlp.org/site/misc/terms.xhtml). After 12 months, it is available under a Creative Commons License (Attribution-NonCommercial 4.0 International), as described at http://creativecommons.org/licenses/by-nc/ $4.0 \%$. 
divided into different groups and allowed to copulate with sexually receptive females, either bearing the lemon scent or no scent. Males who were exposed to the lemon scent ejaculated faster only with females who bore the scent compared to unscented females (Fillion and Blass 1986).

Early experience with neutral odors that predict sexual reward can generate a preference for partners bearing those cues (Kippin et al. 1998; Coria-Avila et al. 2005; Ismail et al. 2009). Kippin et al. (1998) trained males to copulate with sexually receptive females bearing an almond scent. Finally, males were given an open field test to evaluate their preference in which they were given the choice to copulate with two sexually receptive females, one scented and the other unscented. Males ejaculated preferentially with females bearing the scent, thus displaying a conditioned ejaculatory preference (CEP) for the familiar cue. Similarly, other studies have shown that the odor can also be made aversive depending on the contingencies of conditioning. For example, Kippin et al. (1998) also trained males with both receptive unscented females and nonreceptive scented females. Unlike males who were paired with scented females, males trained to associate the odor with nonreceptive females avoided scented females and displayed a CEP for the unscented female. A third group where the odor was randomly paired with receptive and nonreceptive scented females displayed no preference for either of the females.

The rewarding associations fostered during conditioning can come from multiple sources, including sensory stimuli and behaviors aimed at acquiring partners or sexual reward. These associations have been well documented in the context of sexual behavior (Crawford et al. 1993). For instance, Tenk et al. (2009) demonstrated that sexually naïve male rats developed a conditioned place preference toward a particular side of a chamber when this was paired with either intromissions or ejaculations. However, when males were sexually experienced, they developed a conditioned place preference only when that side was paired with ejaculation. Furthermore, Kippin and Pfaus (2001a) trained males allowing them to copulate until reaching five intromissions, one ejaculation without PEI, or one or two ejaculations plus the first intromission following their PEI. Findings showed that only males who achieved one or two ejaculations and were allowed to spend their PEI around the scented female developed a significant CEP toward the scent. In a different experiment, males were allowed to remain in the presence (without access) of a scented female during their PEI after a previous copulatory session with unscented females. Likewise, only males develop a CEP when they achieved one or two ejaculations, but not after five intromissions. These results clearly show that not only ejaculation was necessary to establish a CEP, but also that it is during the post-ejaculatory interval (PEI) when this association takes place.

Altogether, it is clear that early experiences can modulate partner preferences via Pavlovian associations between discrete partner-related cues that predict a sexual reward state. Yet, it is not clear whether latent inhibition will occur as a result of preexposure to the cue alone. The present study evaluated this by preexposing sexually naïve rats to the almond odor either once or five times prior to 10 trials of conditioning where the odor was paired with the post-ejaculatory reward state.

\section{Materials and Methods}

\section{Subjects}

\section{Males}

Ninety-four Long-Evans rats were sexually naïve and weighing $\sim 250 \mathrm{~g}$ at the beginning of the experiment. They were housed in groups of four and two in Plexiglas cages with ad lib access to water and food (Purina Rat Chow). Males were obtained from Charles
River Canada (St-Constant) and kept in a $12 \mathrm{~h}$. reversed lightdark cycle in a room at $21^{\circ} \mathrm{C}$.

\section{Females}

One hundred and twenty Long-Evans rats sexually naïve and weighing $200 \mathrm{~g}$ at the beginning of the experiment were obtained from the same distributor and housed in pairs in the same conditions as males. Females were ovariectomized via bilateral lumbar incisions under ketamine $(50 \mathrm{mg} / \mathrm{mL}) /$ xylazine $(4 \mathrm{mg} / \mathrm{mL})$ anesthesia, mixed at a ratio of $4: 3$, respectively, approximately two weeks before the beginning of the experiment. Sexual receptivity was induced by subcutaneous injections of $10 \mu \mathrm{g}$ estradiol benzoate (Steraloids, injected sc in $0.1 \mathrm{~mL}$ of sesame oil) $48 \mathrm{~h}$ prior to each training session, and $500 \mu \mathrm{g}$ of progesterone (Steraloids, injected sc in $0.1 \mathrm{~mL}$ of sesame oil) $4 \mathrm{~h}$ prior to each training session. Stimulus females were scented with $0.6 \mathrm{~mL}$ of pure almond extract (Blue Ribbon), split equally in the back of their neck and anogenital region as previously done by Kippin and Pfaus (2001b). Different females were assigned to each male randomly for every training session.

\section{Apparatus}

All conditioning sessions were conducted in Plexiglas unilevel pacing chambers $(38 \times 60 \times 38 \mathrm{~cm})$ with bedded floors and bisected by a transparent Plexiglas divider with one-hole large enough for the female to cross, but not the male, as it has been previously found that pacing copulation where males have restricted access to a family facilitates the development of a CEP (Ismail et al. 2009). The cage bedding was not changed between conditioning sessions, and animals trained with scented females were trained in separate cages and rooms from the ones trained with unscented females. The final copulatory preference test took place in a large open field $(123 \times 123 \times 46 \mathrm{~cm})$ filled with clean bedding. All sessions were recorded and subsequently scored with using a behavioral scoring program (Cabilio 1996) that counted frequencies and latencies of individual sexual behaviors (e.g., mounts, intromissions, and ejaculations; as in Sachs and Barfield 1976; Pfaus et al. 1990; Meisel and Sachs 1995).

\section{Procedure}

The common procedure of the experiment is depicted in Figure 1.

\section{Groups}

Males were assigned and equally divided into one of three main groups: control (no preexposure), one trial of preexposure, or five trials of preexposure. The control group was divided into two subgroups: half of these males were trained with sexually receptive scented females $(\mathrm{ScF})$, whereas the other half was trained with sexually receptive unscented females (UnScF). The experimental animals were divided into two groups: males who were preexposed to the odor one time (1t), and males who were preexposed five times $(5 \mathrm{t})$; each of these groups was further divided into two different conditions: trained with ScF or trained with UnScF.

\section{Context preexposure}

All animals were exposed five times to the training chamber on a daily basis for $30 \mathrm{~min}$ prior to the preexposure phase (or the training phase in the case of the control group), in order to habituate them to the training environment, as it has been shown that a novel environment disrupts copulation in sexually naïve rats (Pfaus and Wilkins 1995).

\section{CS preexposure (latent inhibition procedure)}

CS preexposure trials consisted of animals being placed in one side of the chamber with an almond-soaked gauze pad placed in the other side for $30 \mathrm{~min}$. Preexposure trials occurred at a 4-d interval, whereas control groups remained in their home cage until the first 


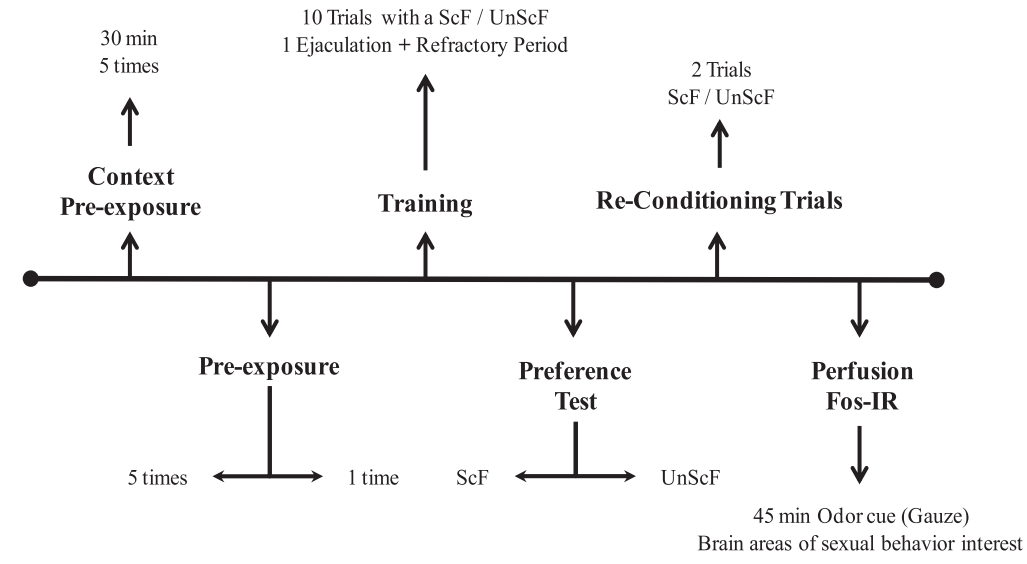

Figure 1. General experimental procedure.
$250 \mathrm{~mL}$ of ice cold $4 \%$ paraformaldehyde. Brains were extracted and post-fixed in clean $4 \%$ paraformaldehyde for $4 \mathrm{~h}$, to be later on stored for $36 \mathrm{~h}$ in a $30 \%$ sucrose solution. Finally, the brains were frozen, covered in aluminum foil and stored at $-80^{\circ} \mathrm{C}$.

\section{Fos immunohistochemistry $(\mathrm{IHC})$ and analysis}

IHC was conducted as in previous studies (e.g., Kippin et al. 2003). Coronal brain sections were incubated sequentially with $30 \% \mathrm{w} / \mathrm{w}$ hydrogen peroxide $\left(\mathrm{H}_{2} \mathrm{O}_{2}\right)$ in Tris-buffered saline (TBS) for $30 \mathrm{~min}$ at room temperature, 3\% normal goat serum (NGS) in .05\% Triton TBS for $90 \mathrm{~min}$ at $4^{\circ} \mathrm{C}$, rabbit polyclonal anti-Fos (Oncogene Science; diluted 1:75,000) in $0.05 \%$ Triton TBS with $3 \%$ NGS for $72 \mathrm{~h}$ at $4^{\circ} \mathrm{C}$, biotinylated goat antirabbit IgG (Vector Laboratories; 1:200)

conditioning trial. Animals in the $1 \mathrm{t}$ group were preexposed when the $5 t$ group was preexposed for the fifth time.

\section{Conditioning}

Following the preexposure phase, all animals were trained to develop a CEP for a sexually receptive female with or without bearing an almond odor (depending on group membership), using a similar procedure to the one described in Kippin and Pfaus (2001b). All males were given 10 training conditioning trials at 4-d intervals during the middle third of the dark phase of the light-dark cycle.

During each sexual behavior conditioning trial, males were placed into the chamber for $5 \mathrm{~min}$ prior to receiving a sexually receptive female (scented or unscented depending on the group). The pair was allowed to copulate freely until ejaculation was achieved, and the test was terminated once the male mounted the female after his post-ejaculatory refractory period had dissipated. Thus, although in previous studies rats were allowed to copulate for $30 \mathrm{~min}$ (to multiple ejaculations) in order to develop a CEP (e.g., Kippin and Pfaus 2001b), here only one ejaculation was used as the criterion considering that the numbers of ejaculation reached in $30 \mathrm{~min}$ and the ejaculation latency varies greatly across male rats during their first sexual experiences. Kippin et al. (2001) established that ejaculation creates a rewarding state and that male must have experienced the presence of the scented female during the refractory period for a CEP for this female to occur. Therefore, by allowing males to remain with the females after one ejaculation only, we equated the induction of this rewarding state in males across the groups.

\section{Copulatory preference test}

Four days after the last sexual behavior conditioning trial, each male was placed in the open field and allowed to explore for 5 min. Subsequently, two females, one ScF and one UnScF, were placed simultaneously into the open field both equally distant from the male. Males were allowed to copulate freely with either female for $30 \mathrm{~min}$. The test was video recorded and scored subsequently for the different sexual behaviors (mounts, intromissions, and ejaculations) that each female received from the male.

\section{Perfusion}

Following the preference test, males were given two more training trials at 4-d intervals exactly as their training conditions. Four days after, males were exposed for $40 \mathrm{~min}$ to $1 \mathrm{~mL}$ of the almond odor alone on a gauze pad on the other side of the pacing chamber. Subsequently, males were injected with sodium pentobarbital (Euthanyl, $120 \mathrm{mg} / \mathrm{kg}$, i.p.) and perfused intracardially with $250 \mathrm{~mL}$ of ice-cold phosphate buffered saline (PBS) followed by in $0.05 \%$ Triton TBS with $3 \%$ NGS for $1 \mathrm{~h}$ at $4^{\circ} \mathrm{C}$, and avidin-biotinylate-peroxidase complex (Vectastain Elite ABC Kit, Vector Laboratories; diluted 1:55) for $2 \mathrm{~h}$ at $4^{\circ} \mathrm{C}$. Sections were washed in TBS (35 $\mathrm{min})$ between each incubation. Immunoreactions were stained by sequential treatments at room temperature with 50-mM Tris for $10 \mathrm{~min}, 3,3^{\prime}$-diaminobenzidine (DAB) in 50-mM Tris $(0.1 \mathrm{~mL}$ of $\mathrm{DAB} /$ Tris buffer, $\mathrm{pH} 7.8)$ for $10 \mathrm{~min}, \mathrm{DAB} / 3 \%$ per $100 \mathrm{~mL}$ of DAB/Tris buffer $\mathrm{H}_{2} \mathrm{O}_{2}$ ). Sections were mounted on gel-coated slides and allowed to dry, then dehydrated, cleared in Hemo-D, coverslipped, and examined under a microscope.

Brain sections were examined at $40 x$, and the number of Fos-positive cells was counted bilaterally from each region from five different sections per rat using a Leitz Microscope connected to a computerized image-analysis system (ImageJ).

Fos-IR was observed in brain regions previously related to sexual behavior and CEP to evaluate the neural activation evoked by the odor alone. The regions examined were the same as those in Kippin et al. (2003), and were defined using the borders in Paxinos and Watson (1998). These included the medial preoptic area (mPOA, $-0.35 \mathrm{~mm}$ from bregma), nucleus accumbens shell and core (NAc Shell and NAc Core, respectively, $1.65 \mathrm{~mm}$ from bregma), piriform cortex (Pir Ctx, $3.70 \mathrm{~mm}$ from bregma), ventromedial hypothalamic nucleus $(\mathrm{VMH},-2.56 \mathrm{~mm}$ from bregma), ventral tegmental area (VTA, $-6.04 \mathrm{~mm}$ from bregma), prefrontal cortex divided into cingulate cortex area $1(\mathrm{Cg} 1,3.70 \mathrm{~mm}$ from bregma), prelimbic (PrL, $3.70 \mathrm{~mm}$ from bregma) and infralimbic (IL, $2.70 \mathrm{~mm}$ from bregma); the basolateral nucleus of the amygdala (BLA, $-2.80 \mathrm{~mm}$ from bregma); and the central nucleus of the amygdala (CeA, $-2.80 \mathrm{~mm}$ from bregma). An average of Fos-positive cells was calculated from three different slides from each rat (five subjects in each group), for each brain area.

\section{Statistical analyses}

A series of mixed design, between-within repeated measures ANOVAs were conducted separately for each copulatory measure (mounts, intromissions, ejaculation, and latency to the first ejaculation), displayed among males in the 4 training groups (1t, $5 \mathrm{t}$, (ScF or UnScF) on the final open-field test. For each significant ANOVA, post-hoc compassions of the means were made using the Tukey HSD correction to ensure to ensure maximal statistical power while correcting for family-wise error. Furthermore, partial eta square $\left(\eta_{p}^{2}\right)$ was calculated as effect size for each comparison. Additionally, a $1 \times 2$ chi square $\left(\chi^{2}\right)$ analysis was conducted for the percentage of first ejaculation choice for each group, and a $2 \times 2 \chi^{2}$ analysis to contrast the ejaculatory preference between the control groups. Furthermore, Cramer's V $(V)$ and Phi $(\phi)$ effect sizes were conducted as effect size for the $1 \times 2$ and $2 \times 2 \chi^{2}$ analyses, respectively. $\mathrm{H}_{2} \mathrm{O}_{2}$ in 50-mM Tris for $10 \mathrm{~min}$, and $8 \%$ nickel chloride $(400 \mu \mathrm{L}$ Control ScF, and Control UnScF) with the two receptive females 
For the Fos-IR results, the mean of Fos-IR positive cells for each brain area was compared separately among the experimental groups using independent samples $t$-test with a Bonferroni correction of the $\alpha$ level to control for the family-wise error (Miller 1966). Since four comparisons were conducted within each brain area, the $\alpha$ level was set at $0.0125(0.05 / 4=0.0125)$ for statistically significant differences. Only in the comparisons between the control groups, the $\alpha$ level was kept at 0.05 , since those independent $t$-tests compared only two groups. Cohen's $d$ effect size statistics were also calculated as a measure of effect size. The following comparisons between groups were conducted for each brain area of interest: $5 t \mathrm{ScF}$ versus 1t ScF, 5t UnScF versus $1 \mathrm{t} \mathrm{UnScF}$, 5t ScF versus $5 t$ UnScF, 1t ScF versus $1 \mathrm{t} \mathrm{UnScF}$, and ScF control versus UnScF control. The latter comparisons were run separately.

\section{Results}

\section{Behavioral analyses}

Two males that did not copulate during the final open field test were not included in the analyses. Although five min of exploration in the open field have previously been used effectively as a period of acclimation for males before the open field test (e.g., Kippin and Pfaus 2001b), it is believed that natural differences in novelty aversion vary in such ways that some animals are affected more than others, and likely show fear responses (such as hugging the walls of an open field) as we observed.

The scores for the different copulatory behaviors by female for all groups during the open field test are depicted in Figure 2. As shown on panels A and B, males did not show consistent differences of mounts for either type of female among groups, whereas males in the control groups displayed a higher number of intromissions toward the female they were trained with. For the mean of mounts, the ANOVA analysis did not detect a statistically significant interaction between Female $\times$ Group, $F_{(5,88)}=0.649, P=$ $0.663, \eta_{p}^{2}=0.036$. As for the mean of intromissions, there was a statistically significant interaction between Female $\times$ Group, $F_{(5,88)}=2.56, P=0.033, \eta_{p}^{2}=0.128$. Post-hoc analyses with Tukey HSD correction revealed that males in the control ScF displayed a marginally higher mean of intromissions for the ScF $(M=18.81)$ over the UnScF $\left(M=15.63, P<0.1, \eta_{p}^{2}=0.024\right)$, whereas males in the control UnScF displayed a statistically significantly higher mean of intromissions for the UnScF $(M=20.5)$ over the ScF $(M=$ $\left.15.69, P<0.05, \eta_{p}^{2}=0.054\right)$. Similarly, males in the $1 \mathrm{t} \mathrm{UnScF}$ that displayed a significantly higher mean of intromissions for the UnScF $(M=18.44)$ over the ScF $\left(M=14.31, P<0.05, \eta_{p}^{2}=0.04\right)$.

As shown on panel $C$ of Figure 2, there was no clear general pattern for the mean of 1st ejaculation choice latency, except for the UnScF control group, where males appeared to take less time to ejaculate first with the familiar female. The ANOVA detected a statistically significant interaction between Female $\times$ Group, $F_{(5,88)}=2.548, P=0.034, \eta_{p}^{2}=0.12$. Post-hoc analyses with Tukey HDS correction revealed that males in the UnScF control group displayed a statistically significantly lower mean latency to ejaculate first with the UnScF $(M=440.63)$ over the ScF $\left(M=1165.13, P<0.001, \eta_{p}^{2}=0.11\right)$, just like males in the $5 \mathrm{t} \mathrm{ScF}$ that displayed a statistically significantly lower mean latency to ejaculate first with the UnScF $(M=606.06)$ over the ScF $(M=$ 1069.93, $\left.P<0.05, \eta_{p}^{2}=0.043\right)$.

As shown on panel D of Figure 2 for the mean ejaculations per female, males displayed a preference to ejaculate more with the familiar female, except for both groups of males in the CS trained with ScF that did not show a preference for either of the females. The ANOVA detected a statistically significant interaction of Female $\times$ Group, $F_{(5,87)}=3.75, P=0.004, \eta_{p}^{2}=0.177$. Post-hoc analyses with the Tukey HSD correction revealed that males in the control UnScF displayed a statistically significantly higher
A
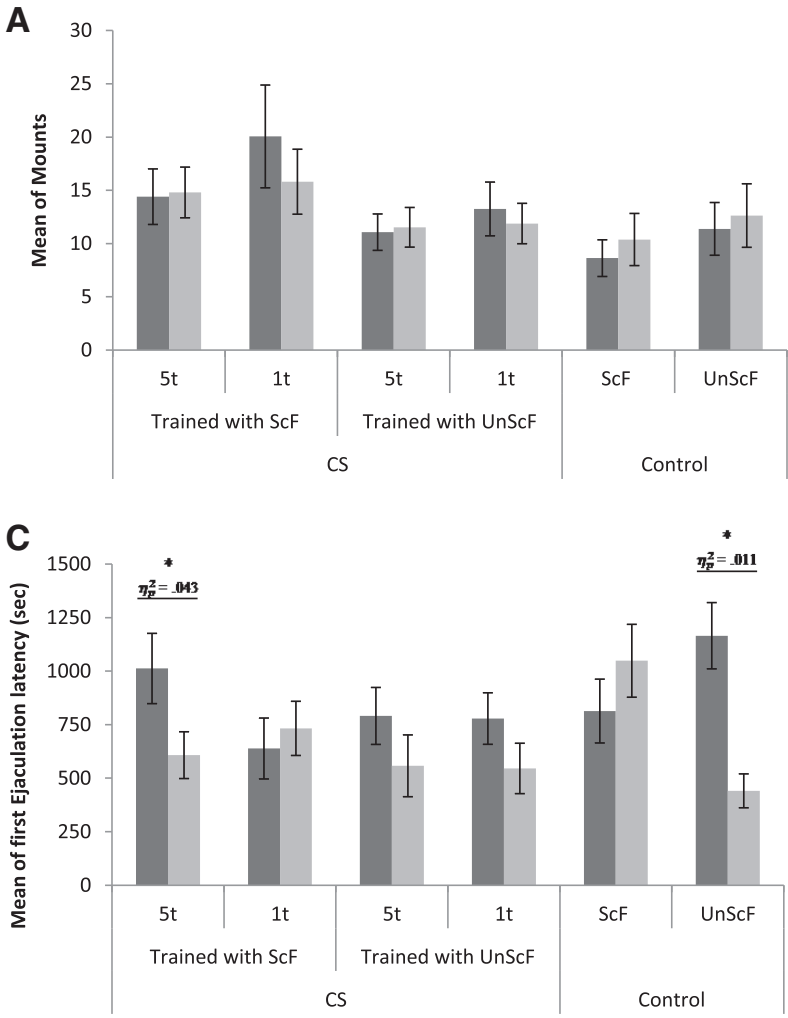
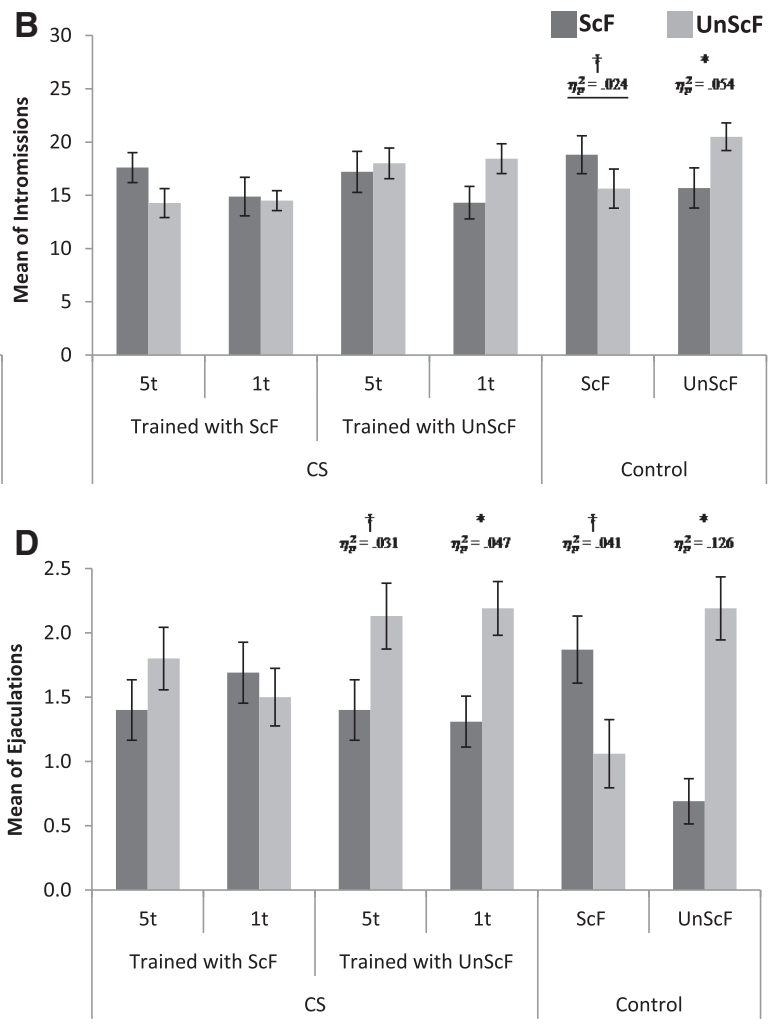

Figure 2. Mean of copulatory behaviors ( \pm SEM) per group during the open field test. $\dagger=P<0.01 ;\left(^{*}\right) P<0.05 ; \eta_{p}^{2}=$ partial eta square. 


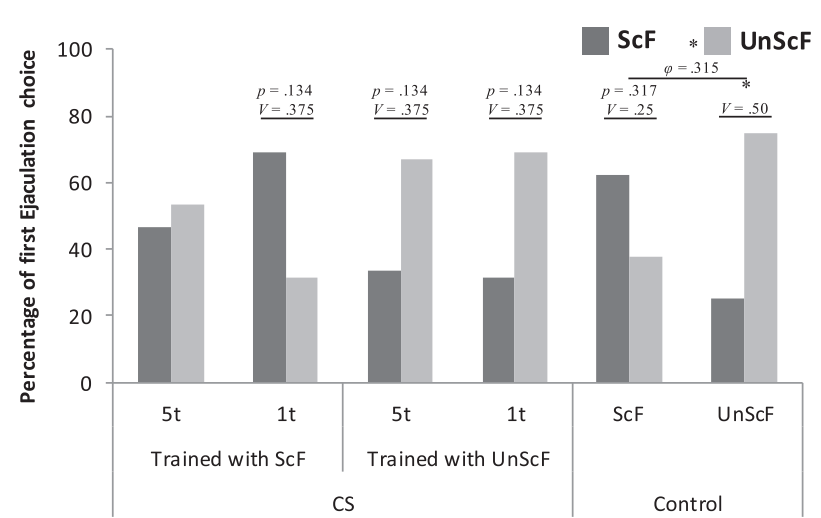

Figure 3. Percentage of 1 st ejaculation choice per group during the open field test. $\left(^{*}\right) P<0.05, V=$ Cramer's V; $\phi=$ phi.

mean of ejaculations for the UnScF $(M=2.19)$ over the ScF $(M=$ $\left.0.687, P<0.001, \eta_{p}^{2}=0.126\right)$, whereas males in the control ScF displayed a statistically marginally higher mean of ejaculations for the ScF $(M=1.63)$ over the UnScF $\left(M=1.06, P<0.1, \eta_{p}^{2}=0.041\right)$. Furthermore, males in the $1 \mathrm{t} \mathrm{UnScF}$ group displayed a statistically significantly higher mean of ejaculations for the UnScF $(M=2.19)$ over the ScF $\left(M=1.31, P<0.05, \eta_{p}^{2}=0.047\right)$, whereas males in the $5 \mathrm{t}$ UnScF displayed a statistically marginally higher mean of ejaculations for the UnScF $(M=2.13)$ over the ScF $(M=1.4, P<$ $0.088, \eta_{p}^{2}=0.031$ ). Neither of the males preexposed to the CS and later on trained with ScF displayed a difference in the mean of ejaculations for either of the females ( $P s>0.05)$.

The percentage of males that chose ScF or UnScF for their first ejaculation is shown in Figure 3. Males in all groups, except in the $5 \mathrm{t} \mathrm{ScF}$ group, clearly preferred the female they were trained with to ejaculate with first. No statistically significant differences were found in percentage of first ejaculation choice for the 5t ScF, $\chi^{2}(1)=0.067, P=0.796, V=0.067$. Statistically marginal differences were found for the $5 \mathrm{t} \mathrm{UnScF}, \chi^{2}(1)=2.25, P=0.134, V=$ 0.375 ; in the $1 \mathrm{t} \mathrm{ScF}, \chi^{2}(1)=2.25, P=0.134, V=0.375$; and $1 \mathrm{t}$ UnScF, $\chi^{2}(1)=2.25, P=0.134, V=0.375$. Statistically significant differences were found for the UnScF control, $\chi^{2}(1)=4.0, P=$ $0.046, V=0.50$, yet no statistically significant differences were found in the ScF control, $\chi^{2}(1)=0.60, P=0.317, \quad V=0.25$. However, a $2 \times 2 \chi^{2}$ analysis between the two control groups revealed that, overall, they preferred different females to ejaculate first with, $\chi^{2}(1)=4.571, P=0.033, \phi=0.315$.

\section{Fos-IR analyses}

Figures 4 and 5 show an example of the Fos-IR in each of the brain area of interest for each of the groups. The mean number of Fos-IR positive cells ( \pm SEM) for each group in the brain areas of interest are presented in Table 1. Following exposure to the odor cue in the group trained with $\mathrm{ScF}$, the 1t group had a higher mean of Fos positive cells than the $5 t$ group in the mPOA, NAc Core, BLA, and VTA, whereas the $1 \mathrm{t}$ had a lower activation than the ScF control group in the NAc Core and Shell, and BLA, yet a higher activation in the mPOA. Conversely, following exposure to the odor cue in the group trained with UnScF, the 5t groups had a higher activation than the 1t group in the NAc Core and Shell, BLA and CeA, whereas the $5 t$ group had a lower mean of Fos positive cells than the ScF control group in the VTA, NAc Core and Shell, yet higher than the ScF control group in the CeA. The reliability of these observations was partially confirmed by t test with a Bonferroni correction and $d$ effect sizes.

\section{mPOA}

No statistically significant differences were found comparing males in the $5 \mathrm{t}$ ScF versus $1 \mathrm{t} \mathrm{ScF}$, although the effect size revealed a difference of a high magnitude, $t_{(8)}=-1.68, P>0.05, d=2.381$; males from the $5 \mathrm{t}$ UnScF versus $1 \mathrm{t}$ UnScF groups, $t_{(8)}=0.092, P>$ $0.05, d=1.308$; males in the $5 \mathrm{t} \mathrm{ScF}$ versus $5 \mathrm{t} \mathrm{UnScF}, t_{(8)}=-1.52$, $P>0.05, d=2.148$; nor between males from the $1 \mathrm{t}$ ScF versus $1 \mathrm{t}$ UnScF groups, $t_{(8)}=1.33, P>0.05, d=1.888$.

\section{VTA}

No statistically significant differences were found comparing males in the $5 \mathrm{t} \mathrm{ScF}$ versus $1 \mathrm{t} \mathrm{ScF}$, although the effect size revealed a difference of a high magnitude, $t_{(8)}=-1.68, P>.05, d=2.057$; males from the $5 \mathrm{t}$ UnScF versus $1 \mathrm{t}$ UnScF groups, $t_{(8)}=0.092, P>0.05, d=2.98$; not between males in the $5 \mathrm{t} \mathrm{ScF}$ versus $5 \mathrm{t}$ UnScF groups, $t_{(8)}=$ $-1.52, P>0.05, d=1.17$; nor between males from the $1 \mathrm{t}$ ScF versus $1 \mathrm{t}$ UnScF groups, although the effect size reveals a difference of a high magnitude, $t_{(8)}=1.33, P>0.05, d=2.307$.

\section{NAc shell}

No statistically significant differences were found comparing males in the $5 \mathrm{t} \mathrm{ScF}$ versus $1 \mathrm{t} \mathrm{ScF}, t_{(8)}=0.58, P>0.05, d=0.357$; not between males from the $5 \mathrm{t} \mathrm{UnScF}$ versus $1 \mathrm{t} \mathrm{UnScF}$ groups, although the effect size revealed a difference of a high magnitude, $t_{(8)}=1.83$, $P>0.05, d=2.592$; not between males in the $5 \mathrm{t}$ ScF versus $5 t$ UnScF, although the effect size also revealed a difference of a high magnitude, $t_{(8)}=-2.29, P>0.05, d=3.24$; nor between males from the $1 \mathrm{t} \mathrm{ScF}$ versus $1 \mathrm{t}$ UnScF groups, $t_{(8)}=0.42, P>0.05, d=0.599$.

\section{NAc core}

No statistically significant differences were found comparing males in the 5t ScF versus $1 \mathrm{t} \mathrm{ScF}, t_{(8)}=-1.16, P>0.05, d=1.649$; not between males from the $5 \mathrm{t} \mathrm{UnScF}$ versus $1 \mathrm{t} \mathrm{UnScF}$ groups, $t_{(8)}=1.48, P>0.05, d=2.096$; not between males in the 5t ScF versus $5 t$ UnScF groups, although the effect size also revealed a difference of a high magnitude, $t_{(8)}=-1.89, P>-0.05, d=2.676$; nor between males from the $1 \mathrm{t} \mathrm{ScF}$ versus $1 \mathrm{t} \mathrm{UnScF}$ groups, $t_{(8)}=0.99$, $P>0.05, d=1.393$.

\section{BLA}

No statistically significant differences were found comparing males in the $5 \mathrm{t} \mathrm{ScF}$ versus $1 \mathrm{t} \mathrm{ScF}, t_{(8)}=1.03, P>0.05, d=1.453$; not between males from the $5 \mathrm{t}$ UnScF versus $1 \mathrm{t}$ UnScF groups, $t_{(8)}=1.4, P>0.05$, $d=1.986$; not between males in the $5 \mathrm{t} \mathrm{ScF}$ versus $5 \mathrm{t}$ UnScF groups, $t_{(8)}=-1.09, P>0.05, d=0.845$; nor between males from the $1 \mathrm{t} \mathrm{ScF}$ versus $1 \mathrm{t} U \mathrm{UnScF}$ groups, $t_{(8)}=0.05, P>0.05, d=0.088$.

\section{CeA}

No statistically significant differences were found comparing males in the $5 \mathrm{t}$ ScF versus $1 \mathrm{t} \mathrm{ScF}, t_{(8)}=1.25, P>0.05, d=1.768$; nor between the $5 t$ UnScF versus $1 t$ UnScF groups, although the effect size magnitude is deemed large, $t_{(8)}=1.93, P>0.05, d=2.725$; not between males in the $5 \mathrm{t} \mathrm{ScF}$ versus $5 \mathrm{t}$ UnScF groups, $t_{(8)}=-0.69$, $P>0.05, d=1.543$; nor between males from the $1 \mathrm{t}$ ScF versus $1 \mathrm{t}$ UnScF groups, $t_{(8)}=-0.26, P>0.05, d=0.375$.

All the nonreported comparisons did not reach statistical significant differences $(P \mathrm{~s}>0.05)$ along with negligible $d$ effect sizes $(d s<1.0)$.

As shown in Table 2 in the companion manuscript, following exposure to the odor cue, males in the ScF control group had a higher mean of Fos-IR than the UnScF control group in all brain areas, except in the CeA. The reliability of these observations and 

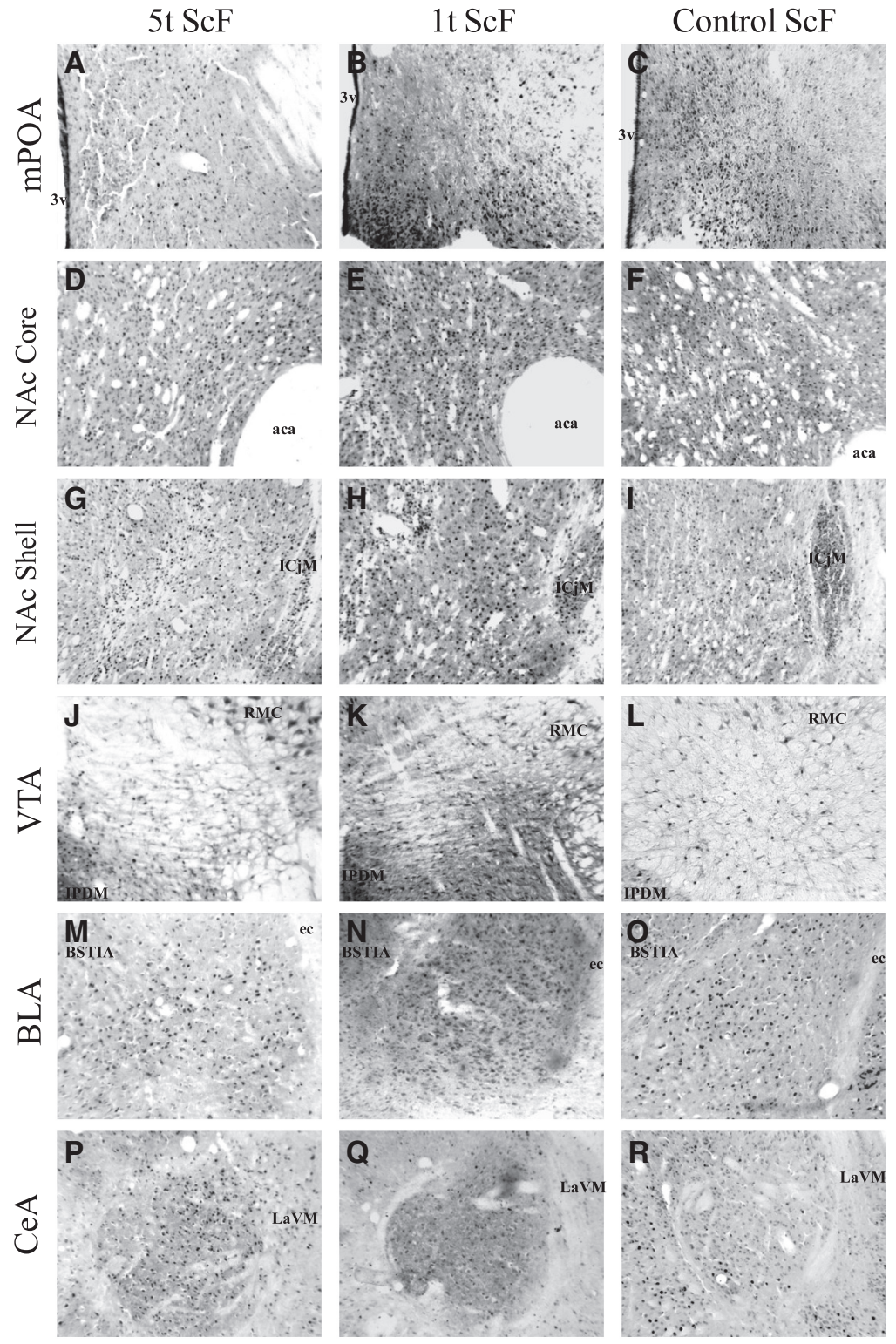

Figure 4. Fos immunoreactivity (Fos-IR) following exposure to the sexually conditioned odor before perfusion in males preexposed five times to the odor before being conditioned with scented females (5t ScF), in males preexposed one time before being conditioned with scented females (1t ScF), and control paired males conditioned with scented females (Control ScF), in brain areas of interest. Pictures were taken accordingly to Paxinos and Watson (1998) in the medial preoptic area (mPOA): $\pm-0.40 \mathrm{~mm}$ from Bregma $(A-C)$; Nucleus accumbens Core and Shell (NAc Core/Shell): $\pm 1.70 \mathrm{~mm}$ from Bregma (core: $D-F$, shell: $G-l)$; Ventral tegmental area (VTA): $\pm-6.04 \mathrm{~mm}$ from Bregma $(J-L)$; the Basolateral amygdala (BLA): $\pm-3.14 \mathrm{~mm}$ from Bregma $(M-O)$, and in the Central nucleus of the amygdala (CeA): $\pm-2.80 \mathrm{~mm}$ from Bregma $(P-R)$. Abbreviations used in the figure: $(3 \mathrm{v}) 3 \mathrm{rd}$ ventricle, (aca) anterior commissure, (ICjM) islands of Calleja major islands, (RMC) magnocellular part of red nucleus, (IPDM) dorsomedial interpeduncular nucleus, (ec) external capsule, (BSTIA) intraamigdaloid division of the bed nucleus of the stria terminalis, (LaVM) ventrolateral part of the lateral amagindaloid nuleus. See Table 1 for mean \pm S.E.M. and text for statistical comparisons.

details of the results are provided in the companion manuscript, Quintana et al. 2018.

\section{Discussion}

The present study examined whether latent inhibition could develop to a neutral olfactory cue used in the development of
CEP in males for a female bearing the cue. Previous studies have found that single CSs and complex contextual cues can become latently inhibited to modulate sexual arousal and CEP (e.g., Kippin et al. 2001; Kippin and Pfaus 2001a,b). In the present study, male rats preexposed five times to the odor cue before being trained with ScF (5t ScF group) did not developed a CEP for the ScF, unlike when males were preexposed five times to the odor and subsequently trained with UnScF (5t UnScF group), or when preexposed only once to the odor (1t ScF and 1t UnScF groups). Furthermore, the Fos-IR analysis regarding the odor exposure demonstrated a differential pattern of activation depending on the odor preexposure and the conditioning contingencies. Namely, a general decrement in Fos-positive cell counts was found as the number of preexposure trials increased compared to the control ScF group. These results show that five trials of preexposure of the neutral almond odor alone before pairing the odor with the post-ejaculatory reward state disrupts the development of CEP, whereas one trial does not. This latent inhibition at the behavioral level was accompanied by significantly fewer Fos positive cells activated by the odor alone within regions of the brain that have been shown previously to be activated by the odor alone following training for CEP (Kippin et al. 2003).

\section{Behavioral analyses}

Latent inhibition refers to a disruption in associative strength when a neutral cue used as a CS is preexposed alone prior to conditioning (e.g., Lubow and Moore 1959). Regardless of the paradigm, latent inhibition is reflected in the reduction of novelty in the CS, leading to a slow rate of acquisition of excitation or inhibition, and a small conditioned response (Schmajuk 2002). Thus, it can be reasoned that the novelty of the odor was reduced enough to impair the development of CEP after 5 preexposure experiences. These data are reminiscent of reports in which the degree of latent inhibition depends on the number of trials of preexposure (Lantz 1973; Zamble et al. 1986). Latent inhibition has also been shown to be context-specific phenomenon (i.e., Wickens et al. 1983). Interestingly, although the training and testing phases were conducted in two different contexts, the latent inhibition effect found in the 5t ScF group remained the same regardless of this change in context. This can be explained because all males were preexposed to the training context before the preexposure phase. Latent inhibition is not context-dependent if the training context is preexposed before the CS preexposition phase (McLaren et al. 1994). Like other type of CSs, a context is 

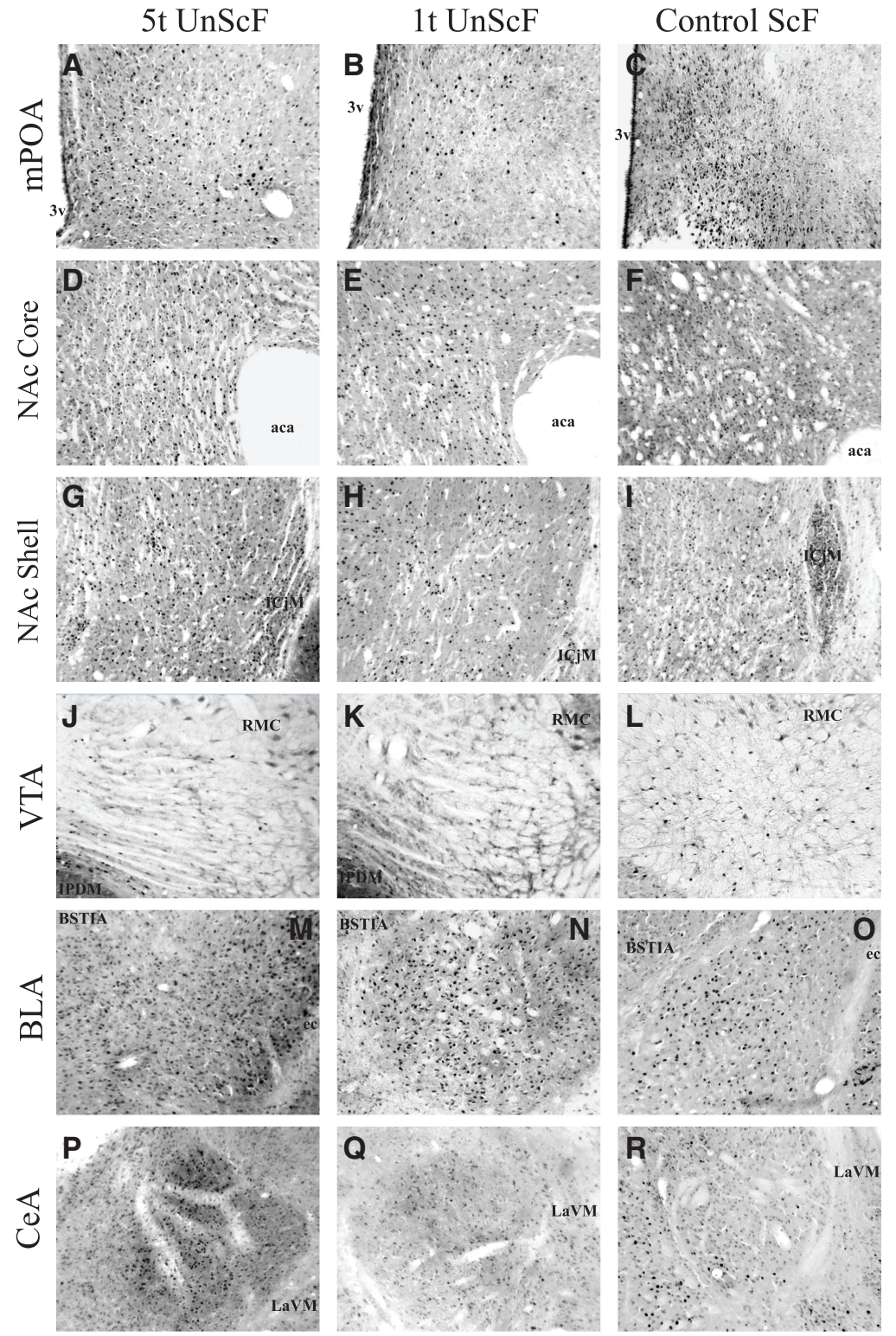

Figure 5. Fos immunoreactivity (Fos-IR) following exposure to the sexually conditioned odor before perfusion in males preexposed five times to the odor before being conditioned with unscented females (5t UnScF), in males preexposed one time before being conditioned with unscented females (1t UnScF), and control paired males conditioned with scented females (Control ScF), in brain areas of interest. Pictures were taken accordingly to Paxinos and Watson (1998) in the medial preoptic area (mPOA): $\pm-0.40 \mathrm{~mm}$ from Bregma $(A-C)$; Nucleus accumbens Core and Shell (NAc Core/Shell): $\pm 1.70 \mathrm{~mm}$ from Bregma (core: $D-F$, shell: $G-l$ ); Ventral tegmental area (VTA): $\pm-6.04 \mathrm{~mm}$ from Bregma $(J-L)$; the Basolateral amygdala (BLA): $\pm-3.14 \mathrm{~mm}$ from Bregma $(M-O)$, and in the Central nucleus of the amygdala $(\mathrm{CeA}): \pm-2.80 \mathrm{~mm}$ from Bregma $(P-R)$. Abbreviations used in the figure: (3v) 3rd ventricle, (aca) anterior commissure, (ICjM) islands of Calleja major islands, (RMC) magnocellular part of red nucleus, (IPDM) dorsomedial interpeduncular nucleus, (ec) external capsule, (BSTIA) intraamigdaloid division of the bed nucleus of the stria terminalis, (LaVM) ventrolateral part of the lateral amagindaloid nuleus. See Table 1 for mean \pm S.E.M. and text for statistical comparisons.

conceptualized as an arrangement of exteroceptive physical elements, and prior exposure to a context like a testing chamber would basically reduce the salience of these elements as they are associated with no direct consequence (e.g., McLaren and Mackintosh 2002).

Finally, previous studies on CEP have associated the CS odor in either an excitatory or inhibitory fashion, after experience with sexually receptive or nonreceptive females, respectively (e.g.,
Kippin et al. 1998). In the present study, however, as a result of the preexposure, it is believed the odor became a less or nonrelevant stimulus due to a reduction of novelty or the lack of attention given to it after association with no consequences during the preexposure phase (e.g., Schmajuk 2010). Hence, we argue that the disruption of the CEP due to the latent inhibition procedure is different from, and not simply less intense than, the inhibition found when Kippin et al. (1998) paired the odor with sexual nonreward.

\section{Fos-IR analyses}

The Fos-IR analysis of the brain areas of interest showed different patterns of activation depending on the amount of preexposure and the type of female the males were trained with. Generally speaking, the differential patters of Fos-IR are most likely due to different levels of sensory activation (Pfaus and Heeb 1997). Therefore, we expected to find lower Fos-IR expression in all groups compared to previous studies of Fos-IR expression regarding a cue associated with sexual reward (e.g., Kippin et al. 2003), because males were allowed to ejaculate only one time per trial, unlike previous studies where they were allowed to ejaculate ad lib within a 30 min test (e.g., Kippin et al. 1998; Ismail et al. 2009). This effect was observed most notably in the $5 \mathrm{t}$ groups.

Figure 4 shows the Fos-IR following exposure to the sexually conditioned odor before perfusion in the ScF $5 t$ and 1t groups, and control ScF groups in the brain areas of interest. Preexposing the odor cue before conditioning in the ScF groups lead to a lower activation of the $5 t$ group compared to the $1 \mathrm{t}$ group in the mPOA, NAc Core, BLA, and VTA, whereas the 1t group had a lower Fos-IR compared to the control ScF group in the BLA, NAc Core and Shell. These brain areas have been described previously as part of the neural system responsible for olfactory conditioning in the rat (Pfaus et al. 2012). The pathways are constituted by three main interactive systems that process the olfactory cue or CS, the sexual reward or US, and the integration of both, including brain regions like the $\mathrm{mPOA}$, VTA, NAc Core and Shell, the amygdala, $\mathrm{VMH}$, and the arcuate nucleus of the hypothalamus. These regions are activated unconditionally by olfactory/pheromonal or genitosensory stimulation during copulation (Pfaus and Heeb 1997). In the present study, the conditioned odor likely activated the representation of the sexual reward state, consistent with Pavlov's (1927) account of cortical processing of CSs. Thus, previous findings have shown a higher Fos-IR count in males trained with ScF after the presentation of the paired odor in the Pir Ctx, BLA, 
Table 1. Fos-IR induction by odor cue $(M \pm S E M)$ for each group in the brain areas of interest

\begin{tabular}{|c|c|c|c|c|c|c|}
\hline Brain areas & 5t Trained with ScF & It Trained with ScF & 51 Trained with UnScF & It Trained with UnScF & Control ScF & Control UnScF \\
\hline $\mathrm{mPOA}$ & $88.4 \pm 48.1$ & $320.7 \pm 129.3$ & $210.1 \pm 64.1$ & $139.1 \pm 42.2$ & $127 \pm 20.2$ & $82.3 \pm 13.1$ \\
\hline VTA & $1.5 \pm 1.2$ & $10.4 \pm 6$ & $28 \pm 1$ & $0.6 \pm 0.3$ & $17.6 \pm 7.3$ & $8.3 \pm 2$ \\
\hline NAc Shell & $16.9 \pm 12.4$ & $20.3 \pm 5.3$ & $103.7 \pm 35.8$ & $28.9 \pm 19.6$ & $161.6 \pm 14.3$ & $101.3 \pm 13.2$ \\
\hline NAc Core & $3.3 \pm 1.9$ & $62 \pm 50.3$ & $53.2 \pm 26.3$ & $11.5 \pm 10$ & $1145 \pm 23.1$ & $103.3 \pm 17.2$ \\
\hline BLA & $12.5 \pm 5.5$ & $5.8 \pm 3.5$ & $18.7 \pm 8.8$ & $5.5 \pm 3.3$ & $21 \pm 7.3$ & $19.6 \pm 2.1$ \\
\hline $\mathrm{CeA}$ & $16.4 \pm 6.8$ & $7.3 \pm 2.6$ & $32.7 \pm 13.3$ & $6.1 \pm 3.7$ & $7.9 \pm 1.8$ & $14.9 \pm 4.2$ \\
\hline VMH & $50.7 \pm 13.8$ & $71.3 \pm 42.6$ & $94.1 \pm 71.9$ & $43.3 \pm 20.3$ & $65.8 \pm 35.1$ & $41.1 \pm 17.8$ \\
\hline Pir Ctx & $250.9 \pm 78.1$ & $189.1 \pm 73.2$ & $197.9 \pm 54.4$ & $154.9 \pm 53.7$ & $170.4 \pm 73$ & $115.4 \pm 38$ \\
\hline Cgl & $100.4 \pm 50.2$ & $99.7 \pm 37$ & $96.9 \pm 42.6$ & $98.5 \pm 48.2$ & $99.6 \pm 32.9$ & $98.7 \pm 49.6$ \\
\hline $\operatorname{PrL}$ & $102.4 \pm 43.4$ & $144.2 \pm 47.3$ & $154.1 \pm 86.8$ & $91.3 \pm 48.7$ & $143.9 \pm 46.5$ & $92.6 \pm 50.6$ \\
\hline IL & $24.6 \pm 15.3$ & $64.1 \pm 29.7$ & $43.9 \pm 25.9$ & $23.6 \pm 11.3$ & $65.9 \pm 31.6$ & $23.3 \pm 11.1$ \\
\hline
\end{tabular}

mPOA, medial preoptic area; NAc Shell, Nucleus accumbens shell; NAc Core, Nucleus acumbens core; VTA, Ventral tegmental area; BLA, Basolateral amygdala; CeA, Central nucleus of the amygdala; VMH, Ventral medial hypothalamus; Pir Ctx, Piriform cortex; Cgl, Cingulate cortex; PrL, Prelimbic cortex; IL, Infralimbic cortex.

and NAc Core, and also a higher Fos-IR in the olfactory bulb, amygdala, mPOA, NAc Core and Shell, and VTA in response to estrous odors (Kippin et al. 2003). Therefore, a lower Fos-IR count in the areas mentioned above for the $5 \mathrm{t}$ and $1 \mathrm{t}$ ScF group compared to the control ScF is consistent with latent inhibition of the neutral odor as a CS.

Fos-IR following $1 \mathrm{t}$ of ScF is consistent with what previous studies have shown for ScF control group Fos-IR for the odor cue alone when it is associated with sexual reward in regions like the BLA and NAcc Core (de Jonge et al. 1992; West et al. 1992; Kippin et al. 2003). In contrast, the number of Fos positive cells in the ScF 1t group was higher in the mPOA compared to the ScF control group. The $\mathrm{mPOA}$ is a brain region that regulates sexual arousal, copulatory responses, and integrates biologically relevant cues (e.g., estrous odors) with sexual reward (see Hull and Rodriguez-Manzo 2009). For instance, lesions have shown to abolish copulation in a wide range of male species, whereas electrical or chemical stimulation has shown to facilitate it (Hull et al. 2006).

Figure 5 shows the Fos-IR following exposure to the sexually conditioned odor before perfusion in the UnScF $5 t$ and $1 t$, and the control ScF groups in the brain areas of interest. Following exposure to the odor cue in the group of males trained with UnScF, the $5 t$ group had a higher number of Fos positive cells than the 1 t group the NAc Core and Shell, BLA, and CeA, yet still lower than the ScF control group in the VTA, NAc Core, and Shell. These results were expected given that the odor would lead to lower Fos positive cells in the UnScF 5t group in comparison to the ScF control group, considering that not only did the males undergo five trials of preexposure to the odor without any consequence, but also because they were trained with $\mathrm{UnScF}$ as predictor of the reward. This may explain why, although the $5 t \mathrm{UnScF}$ had a higher number of Fos positive cells than the $1 \mathrm{t} \mathrm{UnScF}$, it was still lower than the ScF control group in brain areas associated with the control or mediation of appetitive behaviors, attention toward reward-related stimuli, and their own incentive salience (Berridge 2007).

Another interesting result was the higher Fos-IR activation in the UnScF 5t to the ScF control group in the CeA. The amygdala and its subnuclei process affective information from different modalities and are critical for reward- or avoidance-related associative learning (Amunts et al. 2005). Different studies on specific nuclei of the amygdala have shown different roles on the male sexual behavior like copulation, arousal, post-ejaculatory quiescence, among others (see Hull and Rodriguez-Manzo 2009). More recently, the BLA has being identified as a main regulation area for positive and negative associations, where projection from the BLA toward the CeA are at the base for negative conditioning asso- ciations, whereas projections from the BLA toward the NAc are at the base for positive associations (Namburi et al. 2015). It could be that higher CeA Fos-IR in the UnScF $5 t$ group is at the base of a negative association with the odor due to its prolonged exposure compared to the all other experimental groups. However, replication and more specific studies are required to corroborate this hypothesis. Doable-labelling IHC for GABA, glutamate, or other neurotransmitters that may exert an inhibitory action in the $\mathrm{CeA}$, either directly or indirectly, may elucidate this finding.

In conclusion, the present study extends the findings on latent inhibition to the realm of conditioned sexual responses, and demonstrates how the preexposure of a neutral cue before conditioning for a CEP can disrupt the preference otherwise found in the control groups. These findings indicate that the preexposure results in a subsequent impairment of the association that links the odor cue to sexual reward and preference, leading to a differential pattern of neural activation compared to a control group given the amount of preexposure and the contingencies of training. Thus, how animals establish their preference of a sexual partner appears to be not only an orchestration of their own physiological internal state, but also the behavioral and neural mechanisms during first sexual experiences that follow Pavlovian rules of conditioned associations between the internal reward state that serves as the US and external CSs that predict it.

\section{Acknowledgments}

This research was funded by grants from the Canadian Institutes of Health Research (MOP-74563) to JGP, the Consejo Nacional de Ciencia y Tecnología (Chile) to GRQ, and Fonds Québec de la Reserche en Santé to the Center for Studies in Behavioral Neurobiology. The authors would like to thank Nada Hafez, Amanda Harris, Andres Guizar, Sara Rassi, and Kerstin Wenzel for their valuable work in the data collection. Finally, the first author would like to thank Edgar Vogel for its ideas in the interpretation of the behavioral results, and Cristina McHenry for her insightful corrections of previous versions of this manuscript.

\section{References}

Amunts K, Kedo O, Kindler M, Pierperhoff P, Monholberg H, Shan NJ, Habel U, Schneider F, Zilles K. 2005. Cytoarchitectonic mapping of the human amygdala, hippocampal region and entorhinal cortex: intersubject variability and probability maps. Anat Embryol (Berl) 210: 343-352.

Ayres JJB, Philbin D, Cassidy S, Belling L. 1992. Some parameters of latent inhibition. Learn Motiv 23: 268-287.

Berridge KC. 2007. The debate over dopamine's role in reward: the case for incentive salience. Psychopharmacology 191: 391-431. 
Cabilio S. 1996. Behavioral observation program [unpublished computer software]. Concordia University.

Coria-Avila GA, Ouimet AJ, Pacheco P, Manzo J, Pfaus JG. 2005. Olfactory conditioned partner preference in the female rat. Behav Neurosci 119: $716-725$.

Crawford LL, Holloway KS, Domjan M. 1993. The nature of sexual reinforcement. J Exp Anal Behav 60: 55-66.

de Jonge FH, Tonnaer JA, van Leeuwe H, Tielemans AJ, Louwerse AL, van de Poll NE. 1992. Cerebral glucose utilization during conditioned sexual arousal. Physiol Behav 52: 1009-1013.

Fillion TJ, Blass EM. 1986. Infantile experience with suckling odors determines adult sexual behavior in male rats. Science 231: 729-731.

Hull EM, Rodriguez-Manzo G. 2009. Male sexual behavior. In Hormones, brain and behavior, 2nd ed. (ed. Pfaff DW, Arnold AP, Etgen AM, Fahrbach SE, Rubin RT), Vol. 1, pp. 5-65. Academic Press, San Diego.

Hull EM, Wood RI, McKenna K. 2006. The neurobiology of male sexual behavior. In Knobil and Neill's physiology of reproduction, 3rd ed., (ed. Neill JD), Vol. 2, ch. 33, pp. 1729-1842. Academic Press, San Diego, CA.

Ismail N, Gelez H, Lachapelle I, Pfaus J. 2009. Pacing conditions contribute to the conditioned ejaculatory preference for familiar female in the male rat. Physiol Behav 96: 201-208.

Kendrick KM, Hinton MR, Atkins K. 1998. Mothers determine sexual preferences. Nature 395: 229-230.

Kippin TE, Pfaus JG. 2001a. The development of olfactory conditioned ejaculatory preferences in the male rat I. Nature of the unconditioned stimulus. Behav Brain Res 73: 457-469.

Kippin TE, Pfaus JG. 2001b. The nature of the conditioned response mediating olfactory conditioned ejaculatory preference in the male rat. Behav Brain Res 122: 11-24.

Kippin TE, Talianakis S, Schattmann L, Bartholomew S, Pfaus JG. 1998. Olfactory conditioning of sexual behavior and mate selection in the male rat. J Comp Psychol 112: 389-399.

Kippin TE, Samaha AN, Sotiropoulos V, Pfaus JG. 2001. The development of olfactory conditioned ejaculatory preferences in the male rat II. Parametric manipulation of conditioning session number and duration. Physiol Behav 73: 471-485.

Kippin TE, Cain SW, Pfaus JG. 2003. Estrous odors and sexually conditioned neutral odors activate separate neural pathways in the male rat. Neuroscience 117: 971-979.

Lantz AE. 1973. Effects of numbers of trials, interstimulus interval, and deshabituation on subsequent conditioning in a CER paradigm. Anim Learn Behav 1: 273-277.

Lubow RE, Moore AU. 1959. Latent inhibition: the effect of nonreinforced preexposure of the conditioned stimulus. J Comp Physiol Psychol 52: 415-419.

McLaren IPL, Mackintosh NJ. 2002. An elemental model of associative learning: I. Latent inhibition and perceptual learning. Anim Learn Behav 28: $211-246$.

McLaren IPL, Bennett C, Palisted K, Aitken M, Mackintosh NJ. 1994. Latent inhibition, context specificity, and context familiarity. Q J Exp Psychol B 47: $387-400$.

Meisel RD, Sachs BD. 1995. The physiology of male reproduction. In The physiology of reproduction (ed. Knobil E, Neil JD), Vol. 2, pp. 3-105. Raven, New York.

Miller RG. 1966. Simultaneous statistical inference. Springer.
Namburi P, Beyeler A, Yorozu S, Calhoon GG, Halbert SA, Wichmann R, Holden SS, Mertens KL, Anahtar M, Felix-Ortiz AC, et al. 2015. A circuit mechanism for differentiating positive and negative associations. Nature 520: $675-678$.

Parédez RG, Vazquez B. 1999. What do female rats like about sex? Paced mating. Behav Brain Res 105: 117-127.

Pavlov IP. 1927. Conditioned reflexes: an investigation of the physiological activity of the cerebral cortex (G. V. Anrep, Trans.). Dover, New York.

Paxinos, G, Watson, C. 1998. The rat brain. 4th ed. Academic Press, California.

Pfaus JG, Heeb MM. 1997. Implications of immediate early gene induction in the brain following sexual stimulation of female and male rodents. Brain Res Bull 44: 397-407.

Pfaus JG, Wilkins MF. 1995. A novel environment disrupts copulation in sexually naive but not experienced male rats: reversal with naloxone. Physiol Behav 57: 1045-1049.

Pfaus JG, Mendelson SD, Phillips AG. 1990. A correlational and factor analysis of anticipatory and consummatory measures of sexual behavior in the male rat. Psychoneuroendocrinology 15: 329-340.

Pfaus JG, Kippin TE, Coria-Avila GA, Gelez H, Alfonso VM, Ismail N, Parada M. 2012. Who, what, where, when (and maybe even why)? How the experience of sexual reward connects sexual desire, preference, and performance. Arch Sex Behav 41: 31-62.

Quintana GR, Guizar A, Rassi S, Pfaus JG. 2018. First sexual experiences determine the development of conditioned ejaculatory preference in male rats. LearnMem 25: 522-532.

Rodríguez G, Alonso G. 2002. Latent inhibition as a function of CS intensity in taste aversion learning. Behav Processes 60: 61-67.

Sachs BD, Barfield RJ. 1976. Functional analysis of masculine copulatory behavior in the rat. Adv Study Behav 7: 91-154.

Schmajuk NA. 2002. Latent inhibition and its neural substrates. Kluwer academic publishers, Massachusetts.

Schmajuk NA. 2010. Mechanisms in classical conditioning: a computational approach. Cambridge University Press, Cambridge.

Schnur P, Lubow RE. 1976. Latent inhibition: the effects of ITI and CS intensity during preexposure. Learn Motiv 7: 540-550.

Tenk CM, Wilson H, Zhang Q, Pitchers KK, Coolen L. 2009. Sexual reward in male rats: effects of sexual experience on conditioned place preference associated with ejaculations and intromissions. Horm Behav 55: 93-97.

West CH, Clancy AN, Michael RP. 1992. Enhanced responses of nucleus accumbens neurons in male rats to novel odors associated with sexually receptive females. Brain Res 585: 49-55.

Westbrook RF, Bond NW, Feyer AM. 1981. Short- and long-term decrements in toxicosis-induced odor-aversion learning: the role of duration of exposure to an odor. J Exp Psychol Anim Behav Process 7: 362-381.

Wickens C, Tuber DS, Wickens DD. 1983. Memory for the conditioned response: the proactive effect of preexposure to potential conditioning stimuli and context change. J Exp Psychol Gen 112: 41-57.

Zamble E, Mitchell JB, Findlay H. 1986. Pavlovian conditioning of sexual arousal: parametric and background manipulations. J Exp Psychol Anim Behav Process 12: 403-411.

Received June 26, 2018; accepted in revised form June 29, 2018. 


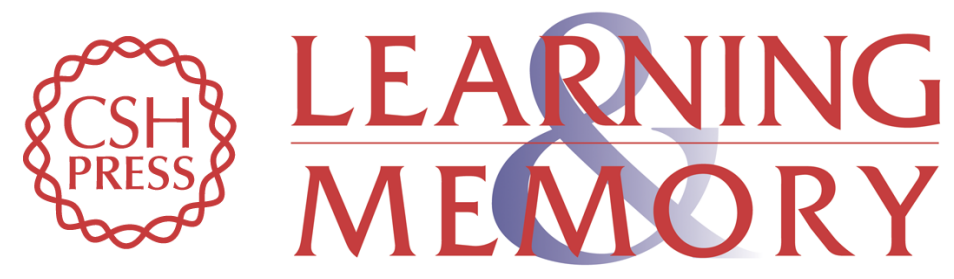

\section{Effect of CS preexposure on the conditioned ejaculatory preference of the male rat: behavioral analyses and neural correlates}

Gonzalo R. Quintana, Misha Jackson, Mojdeh Nasr, et al.

Learn. Mem. 2018, 25:

Access the most recent version at doi:10.1101/lm.048108.118
Related Content First sexual experiences determine the development of conditioned ejaculatory preference in male rats
Gonzalo R. Quintana, Andrés Guizar, Sarah Rassi, et al.
Learn. Mem. October , 2018 25: 522-532
References This article cites 36 articles, 1 of which can be accessed free at:
http://learnmem.cshlp.org/content/25/10/513.full.html\#ref-list-1
Articles cited in:
http://learnmem.cshlp.org/content/25/10/513.full.html\#related-urls
Creative This article is distributed exclusively by Cold Spring Harbor Laboratory Press for the Commons first 12 months after the full-issue publication date (see
License
http://learnmem.cshlp.org/site/misc/terms.xhtml). After 12 months, it is available under a Creative Commons License (Attribution-NonCommercial 4.0 International), as described at http://creativecommons.org/licenses/by-nc/4.0/.
Email Alerting Receive free email alerts when new articles cite this article - sign up in the box at the Service top right corner of the article or click here.

